\title{
Aggressive prevention and preemptive management of vascular complications after pediatric liver transplantation: $A$ major impact on graft survival and long-term outcome
}

\author{
Chiara Grimaldi $^{1}$ | Fabrizio di Francesco ${ }^{2}$ | Fabrizio Chiusolo ${ }^{3}$ (D) | Roberta Angelico ${ }^{1}$ | \\ Lidia Monti $^{3}$ | Paolo Muiesan ${ }^{4}$ | Jean de Ville de Goyet ${ }^{2}$ (D)
}

${ }^{1}$ Department of Pediatric Surgery and Transplantation, Bambino Gesù Children's Hospital, IRCCS, Rome, Italy

${ }^{2}$ Department of Pediatrics and Pediatric Transplantation, ISMETT, UPMC, Palermo, Italy

${ }^{3}$ Pediatric Intensive Care Unit, Bambino Gesù Children's Hospital, IRCCS, Rome, Italy

${ }^{4}$ The Liver Unit, Queen Elizabeth Hospital, Birmingham, UK

\section{Correspondence}

Jean de Ville de Goyet, Department of Pediatrics and Pediatric Transplantation, ISMETT - UPMC, Palermo, Italy.

Email: Jdeville@ismett.edu

\begin{abstract}
Vascular complications are a major cause of patient and graft loss after LTs. The aim of this study was to evaluate the effect of a multimodal perioperative strategy aimed at reducing the incidence of vascular complications. A total of 126 first isolated LTsperformed between November 2008 and December 2015-were retrospectively analyzed. A minimum follow-up period of 24 months was analyzable for 124/126 patients (98.4\%). The aggressive preemptive strategy consisted of identifying and immediately managing any problem and any abnormality in the vascular flow, in any of the hepatic vessels, and at any time after the liver graft revascularization. As a result, with a median follow-up of 57 months (3-112 months), not a single graft has been lost from vascular or biliary problems. The actuarial 8-year graft survival is $96.5 \%$. These results have shown that a combination of technical attention, medical prevention, an early diagnosis, and rapid interventions reduced the negative impact of vascular problems on the outcome of both grafts and patients.

KEYWORDS

children, liver transplantation, outcome, techniques, vascular complications
\end{abstract}

\section{1 | INTRODUCTION}

As a whole, vascular complications are the leading cause of both the early and the late loss of grafts after a liver transplantation. They are associated with high rates of retransplantation and related patient deaths. ${ }^{1-6}$ The incidence is higher in children when compared with adults. This occurs as a consequence of the unique technical challenges that are related to smaller caliber vascular anastomosis, and the (over)sized mismatched grafts used in infants, as well as with a hypoplasia of the PV in children with a biliary atresia. ${ }^{7-14}$

With the intent of decreasing the incidence of vascular problems and graft loss, many pediatric teams have implemented preventive and preemptive management strategies during the last decade, including our center's program. ${ }^{15}$

\section{AIM OF THE STUDY}

This research was an observational retrospective study of all children ( $<18$ years of age) who benefited from a first isolated liver transplantation between November 2008 and December 2015. It was a period of time when a multifaceted strategy for the prevention and the early management of vascular complications had been prospectively implemented (protocol driven, single center, and single

Abbreviations: ALT, alanine aminotransferase; aPTT, activated partial thromboplastin time; CTA, computerized tomography with angiography; ELTR, European Liver Transplantation Registry; GGT, gamma-glutamyltransferase; HA, hepatic artery; ICU, intensive care unit; INR, international normalized ratio; IU, international unit; IVC, inferior vena cava; LT, pediatric liver transplantation; PELD, pediatric end-stage liver disease; POD, postoperative day; PV, portal vein; SD, standard deviation; SPLIT, Studies of Pediatric Liver Transplantation Registry; USD, Doppler ultrasonography. 
team). The objective of the study was to analyze (a) the incidence of vascular complications occurring in the short ( $<1$ month) or late (>1 month) term; (b) their management; and (c) the long-term outcome in terms of graft and patient survival.

\section{3 | MATERIALS AND METHODS}

All of the consecutive first isolated pediatric LTs that were performed at the center between November 1, 2008 (the start of the program), and December 31, 2015, were retrospectively analyzed. One transplant was excluded because the patient had a prolonged cardiac arrest upon reperfusion (cirrhotic cardiopathy) and died within 24 hours and was therefore not analyzable for this study. All of the other patients were selected for the study, and the minimal graft and patient survival was $>3$ months in all of the cases. The data had been previously prospectively collected in the center's database (demographic characteristics, surgical technique, postoperative complications, and their management) and with the consent of the patients.

As per the protocol, all of the patients had received the same induction (anti-interleukin-2 monoclonal antibodies) and maintenance immunosuppression therapy (intent for tacrolimus monotherapy-associated with steroids and/or mycophenolate mofetil, when required by their clinical needs). In addition, as per the protocol, all of the patients were followed perioperatively by implementing a multifaceted strategy aimed at reducing the incidence of vascular complications ${ }^{15-32}$; this latter strategy was used during the whole period of the study and it consisted of 4 main complementary aspects:

\section{1 | Flow-optimizing surgical techniques}

\subsection{1 | Portal vein}

The standard technique for a PV anastomosis was an end-to-end anastomosis; for all of the left lateral segmental grafts, a triangular anastomosis was fashioned between the left donor PV and the recipient PV bifurcation. In all of the recipients with a PV diameter $\leq 5 \mathrm{~mm}$, a longitudinal portal anterior venoplasty was performed, by using a venous vascular graft from the same donor (an iliac vein from the cadaveric donor or an inferior mesenteric vein from the living donor). ${ }^{20}$

\subsection{2 | Hepatic artery}

All anastomoses were performed using microsurgical techniques, and all were performed using 5 times magnification glasses, not with a surgical microscope. A separate-stitch suture with Prolene $8 / 0$ was the standard anastomosis for arteries $\leq 4 \mathrm{~mm}$ in diameter, while a continuous suture with absorbable material was used for the larger ones. As a standard practice, a preference was given to using a short length of a donor artery, with a single anastomosis into a distal recipient site (proper HA, or right or left branch) (Figure 1) ${ }^{21,22}$,

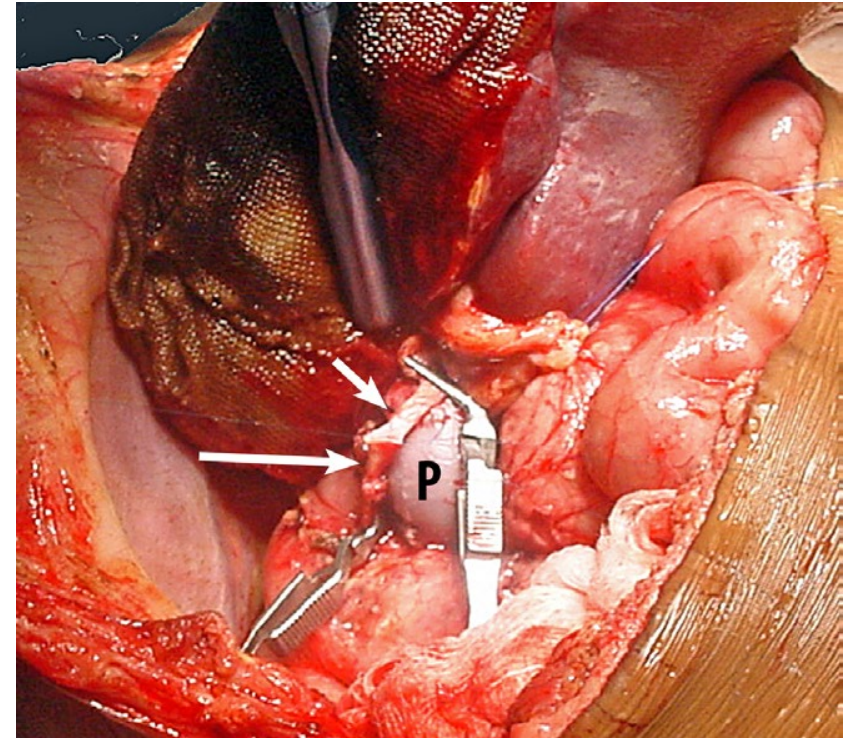

FIGURE 1 Center best practice strategy for a vascular reconstruction at the implantation of a left lateral segment: a short donor artery (left HA) anastomosed into a distal (right HA) recipient by using a microvascular technique and an interrupted suture, a preserved (enlarged if necessary) recipient $P V$ trunk $(P)$, a piggyback large triangular hepatic vein anastomosis, with an adequate size match between the graft volume and the recipient abdomen

the gastroduodenal artery was kept intact unless a division was necessary for dissecting the PV and for reaching the confluence with the splenic and mesenteric veins. When the liver graft was procured with multiple arteries, a back-table reconstruction was performed in the case of a cadaveric procurement, in order to avoid multiple in situ anastomoses; if the graft was from a living donor, the largest graft artery was anastomosed first, with the second (smaller) artery being reconstructed only when a retrograde pulsatile perfusion was absent.

\subsection{3 | Inferior vena cava}

A large triangular piggyback anastomosis was used as a standard for both whole and split grafts. ${ }^{23,24}$ In order to reduce the risk of twisting or of a kinking of the caval anastomosis, with subsequent outflow problems, the small-for-size liver grafts were positioned with care and attached to the anterior abdominal wall by using the falciform ligament.

\subsubsection{Abdominal wall closure}

Attention was given to the closing of the abdomen, so as to prevent a risk of graft compression at the end of the operation. In the case of large-for-size grafts, and in any case where the imaging by USD suggested compression (reduction in flow at attempting closure), abdominoplasty by interposing a prosthesis at the fascia level was carried out, before the mobilized skin flaps were closed over. $^{25,26}$ 


\section{2 | Repeated intraoperative Doppler checks}

USD was performed during all of the transplant procedures, for at least three successive time points: (a) after the arterial reperfusion, (b) at the time of the abdominal closure, and (c) after the closure of the abdomen, before the patient was transferred back into the ICU. In the case of an abnormal Doppler signal (low or absent flow-either in veins or in artery), the graft position and the vascular reconstructions were checked (this included the possibility of reopening the abdomen immediately after the closure), until the problem was solved and until normal Doppler signals were obtained. ${ }^{16,19,27-29,32}$ Blood flow was considered abnormal early after reperfusion if PV flow was $<15 \mathrm{~cm} / \mathrm{s}, \mathrm{HV}$ flow $<10 \mathrm{~cm} / \mathrm{s}$, and HA systolic maximal velocity $<25 \mathrm{~cm} / \mathrm{s}$, or HA resistance index $>0,8$.

\section{3 | Perioperative management of the coagulation profile}

The patient's coagulation was maintained in a balanced status that aimed at avoiding either an excessive coagulopathy (spontaneous or iatrogenic) or a thrombophilic condition (which has been shown to be naturally occurring in first days/weeks after an LT). ${ }^{18,22,30,33}$ The objective was to lower the thrombus formation capacity of the patient, not to achieve a fully active anticoagulation; in that perspective, heparin management was usually not commenced before the second or third day after an LT-a period of time when the patient's INR is usually still $>1.5$. When heparin was started, low doses were used and there was also no aim at achieving any given therapeutic range-with in vitro testingand it was unusual to see, for example, an aPTT $>60$ seconds in these patients. The aim of this "low anticoagulation" was to avoid a post-LT hemorrhage and possible related reinterventions or transfusions.

\section{4 | Intraoperative care}

The coagulation was checked before and during the procedure, aiming at maintaining levels within a range that was lower than normal, but good enough to prevent an excessive blood loss during the procedure, as follows: (a) A slight hypocoagulation state was preferred and this was not corrected, while support was given (fresh frozen plasma) if the INR $>3$; and (b) platelets were transfused only in those patients with an active hemorrhage refractory to conventional surgical hemostasis and with platelets $<50000 / \mu \mathrm{L}$. Hemoglobin level was kept between 7 and $9 \mathrm{gr} / \mathrm{CL}$.

By all (surgical) means, the operations were conducted in order to reduce the blood loss as much as possible. In those rare patients, with a normal coagulation profile before and throughout the procedure (ie, patients with metabolic disease receiving living related liver grafts), a low-dose heparin infusion could be started at the end of the transplant, if the coagulation results remained fully within the normal range at the end of the LT procedure.

For the postoperative course, the following strategy was used:

1. A correction of severe coagulopathy by an infusion of fresh frozen plasma in those cases with an early hepatic dysfunction and/or with an INR $>3$.
2. A supplementation of antithrombin in order to maintain the plasma level around normal values (target: minimal plasmatic value $\pm 15 \%)^{18,22}$

3. The start of an anticoagulation/antiaggregation protocol, when INR was < 1.5:

a. Heparin (5 UI/ $\mathrm{kg} / \mathrm{h}$ intravenous) was started postoperatively when an INR was $<1.5$ and if the platelets were $>50000 / \mathrm{dL}$. This therapy was preferred to any other for the first few days, because it could be more easily suspended if a reintervention is necessary. Heparin was discontinued when the antiaggregant therapy was initiated per os.

b. Aspirin ( $3 \mathrm{mg} / \mathrm{kg} / \mathrm{d}$ orally) and dipyridamole ( $7 \mathrm{mg} / \mathrm{kg} / \mathrm{d}$ orally) therapies were introduced when the patient resumed oral nutrition and at the condition that an INR was $<1.5$ and the platelet count was $>50000 / \mathrm{dL}$. Both of these drugs were discontinued 3 months after the surgery.

For analyzing the follow-up, the following data were collected:

1. The results of USD: This was performed every day for the first 7 days in all of the patients (twice per day in selected cases with intraoperative problems or with complex reconstructions), then twice per week until discharge, and subsequently, bimonthly during the first semester.

2. The early graft functions were assessed by analyzing the blood test results (ALT, total bilirubin, INR, and platelet count) on PODs 1, 2, 3, 5, 7, and 15 .

3. Vascular complications were defined and categorized as follows: absent Doppler signal at USD/established thrombosis at a surgical revision/absent flow found at a conventional angiography/an absence of a contrast enhancement upon a CTA/an abnormal Doppler signal during follow-up showing arterial tardus parvus, or a low flow $(<10 \mathrm{~cm} / \mathrm{s})$ in the PV trunk before the anastomosis.

4. All of the late outcomes were assessed by the graft and patient survival rates (actual, 1 and 5 years posttransplant), as well as by the results at the last follow-up visit (blood levels of ALT, GGT, total bilirubin, and USD). Information about the actual level of immunosuppression (at the last visit) and the last biopsy results (a presence or absence of any signs of ischemic or biliary problems and of acute/chronic rejection or chronic hepatitis, and the Ishak fibrosis score ${ }^{34}$ ) were also collected. Lastly, because vascular problems can cause ischemic damage and, thereby, secondary biliary problems, information was collected about biliary problems in the series, with an attention focused on their type, their management, and their outcome.

Numerical data were expressed by their median values $( \pm S D)$ and/ or by minimum and maximum. The survival rates were calculated as actual rates and then expressed in percentages ( $n$ alive/126). KaplanMeier analyses were used in order to estimate the graft and the patient's late survival. 
TAB LE 1 Demographic data and characteristics of 126 pediatric recipients of a primary isolated liver graft (single-center experience: 2008-2015)

\begin{tabular}{|c|c|c|}
\hline Variable types & Variable subtypes & $\begin{array}{l}\mathrm{N}(\%) \text { or median } \pm \mathrm{SD} \\
\text { (range) }\end{array}$ \\
\hline Age (mo) & & $20.7 \pm 56.1(0.9-225)$ \\
\hline Weight (kg) & & $10.5 \pm 14.7(2.9-78.1)$ \\
\hline Weight <10 kg (N) & & $61(48 \%)$ \\
\hline \multirow{6}{*}{$\begin{array}{l}\text { Indication for } \\
\text { transplantation }\end{array}$} & Biliary atresia & 75 (59.5\%) \\
\hline & Other cholestasis & 12 (9.5\%) \\
\hline & Fulminant failure & $5(4.0 \%)$ \\
\hline & Metabolic disease & $18(14.3 \%)$ \\
\hline & Liver tumors & $13(10.3 \%)$ \\
\hline & Other & $3(2.4 \%)$ \\
\hline \multirow[t]{3}{*}{$\begin{array}{l}\text { PELD at transplant } \\
\text { (score) }\end{array}$} & $\begin{array}{l}\text { Statuses } 1 \text { and } 2 \\
\text { excluded }\end{array}$ & $21 \pm 3.5$ \\
\hline & $\begin{array}{l}\text { Living donor graft } \\
\text { only }\end{array}$ & $18 \pm 0.7$ \\
\hline & $\begin{array}{l}\text { Postmortem donor } \\
\text { graft only }\end{array}$ & $22.5 \pm 9.9$ \\
\hline \multirow[t]{2}{*}{ Donor type } & Living & $38(30.2 \%)$ \\
\hline & Postmortem & 88 (69.8\%) \\
\hline $\begin{array}{l}\text { Donor/recipient } \\
\text { weight ratio }\end{array}$ & & $5.6 \pm 3.6(0.6-15.94)$ \\
\hline \multirow[t]{5}{*}{ Graft type } & Whole liver & $22(17.5 \%)$ \\
\hline & Extended right lobe & $4(3.2 \%)$ \\
\hline & Reduced graft & $6(5.5 \%)$ \\
\hline & Left lateral segment & $88(70.6 \%)$ \\
\hline & $\begin{array}{l}\text { Hyperreduced } \\
\text { (segment II) }\end{array}$ & $6(4.0 \%)$ \\
\hline \multirow{3}{*}{$\begin{array}{l}\text { Parenchymal } \\
\text { division mode }\end{array}$} & In situ & 86 \\
\hline & Ex situ & 10 \\
\hline & $\begin{array}{l}\text { Combined in situ/ } \\
\text { ex situ }\end{array}$ & 8 \\
\hline \multirow{2}{*}{$\begin{array}{l}\text { Cold ischemic time } \\
\text { (min) }\end{array}$} & Postmortem donor & $534 \pm 109$ \\
\hline & Living donor & $205 \pm 91$ \\
\hline
\end{tabular}

\section{4 | RESULTS}

\section{1 | Patient demographics}

During the observation period, 126 first isolated LTs were performed. The demographics and the characteristics of the patients and the grafts are detailed in Table 1.

\section{2 | Vascular reconstructions}

\subsection{1 | PV reconstruction}

PV hypoplasia (diameter $\leq 5 \mathrm{~mm}$ ) was found in $26 / 75$ biliary atresia cases, and a longitudinal anterior venoplasty was performed in all of the 26 cases. One biliary atresia patient benefited from a cavoportal transposition, in order to manage an unusual malformation of the portal system, with an agenesy of the PV. In four patients, a thrombosis of the PV was found (two with a portal cavernoma and two with a partial thrombosis of the PV trunk). Reconstructions were conducted (a) from the superior mesenteric vein and from a large gastric varix in the two patients with a cavernoma; (b) by an allograft substitution in a child with a hepatoblastoma and a PV thrombosis; and (c) by a standard thrombectomy in a teenager with primary sclerosing cholangitis and a PV thrombosis.

\subsection{2 | HA reconstruction}

A single and direct anastomosis was performed in 112 patients (88.9\%), and a double arterial anastomosis was carried out in 14 cases (11.1\%). In all but one patient, the recipient arterial anastomosis site was the proper or common $\mathrm{HA}$; in a single case, the graft was vascularized from the aorta with an aortic conduit (iliac arterial graft from the same donor).

\section{3 | Intraoperative Doppler US findings and management}

As described above, an intraoperative Doppler US was performed in all of the recipients, (a) after a full graft reperfusion; (b) at a closing of the abdominal wall, and (c) before the patient was transferred from the theater back into the ICU.

In 114 patients (90.5\%), the HA flow was adequate at all of the USD checks, intraoperatively, and at follow-up. An immediate perioperative USD showed an abnormal arterial flow in 12 cases (9.5\%) (10 after the reperfusion, one at the closing of the abdomen, and one after the closure). The problems were independent of the graft type (five full-size, five LRD, two split). In five cases, the flow was present, but it was low and the problem was solved by a repositioning of the graft and a splenic artery ligation; all five patients had very large splenomegaly and aged $>3,5$ years. In five cases, the arterial anastomosis was revised with a subsequent correction of the flow. In two cases, graft repositioning allowed for a resolution of the problem. On leaving the operating room, all of the patients had a normal arterial flow at the USD check, but a single one had a lower flow in the right HA that normalized within 24 hours under a heparin therapy. The USD of these patients has been normal at follow-up, in all but one case, who was diagnosed with an arterial stricture 2 months later. This was managed successfully by interventional radiology.

The flow in the PV was normal after the reperfusion in 116 children (92\%). Ten patients (8\%), of whom eight had a PV hypoplasia and longitudinal plasty, had a low PV or an absent flow at the USD check. In five cases, the venous anastomosis was revised (a full redo in one) and the graft was repositioned in order to avoid compression. In four cases, the graft was repositioned, with a prosthetic abdominal closure performed in three cases. One patient had a normal flow in the PV, but with a low flow in the left branch only. This normalized within 24 hours under a heparin therapy. Of 

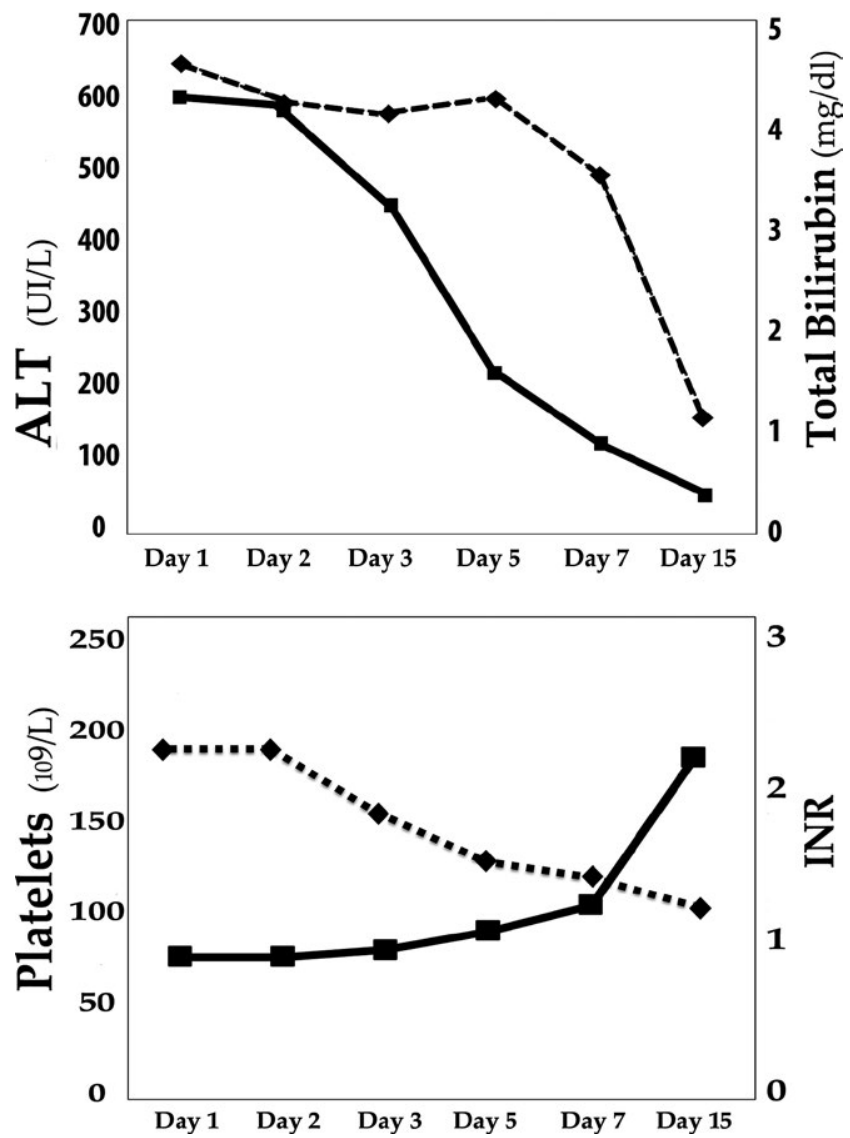

FIGURE 2 The results (median values) of coagulation tests (INR and platelet count) (above) and of liver function tests (total bilirubin and ALT) (below) after 126 pediatric first liver transplantations

the 10 patients with an intraoperative PV problem, all had a normal flow at a later follow-up, but one patient who was diagnosed with a late thrombosis underwent a successful meso-Rex bypass, 2 years after their transplant. No outflow complications were observed in this series.

Overall, on the last operative day of the USD check (performed before the patient was transferred back into the ICU), the flow in all liver vessels was adequate in all of the cases. However, two patients who had a minor abnormality (a low flow in their right HA or in their left PV) were managed by a heparin infusion.

\subsection{Evolution during the first postoperative month and the patient's management}

Six patients had an abnormal arterial flow at their USD check during the first month. A splenic artery steal syndrome was diagnosed in five patients, typically during the first week (days 1-4), and typically also in five children who had severe and long-standing portal hypertension (cirrhotic condition with mean age at transplant $=10$ years): Four patients were treated radiologically (proximal splenic artery embolization) and one was treated by a surgical reexploration with a graft repositioning and a ligation of the splenic artery. ${ }^{31,35}$ Another patient was diagnosed during the third week with an arterial stricture, and this was treated successfully by a percutaneous angioplasty (POD 17). None of these patients had any further problems during their follow-up.

A single patient who was diagnosed with a low PV flow 4 days after their transplant had to be explored surgically: A PV anastomosis was redone and the graft was repositioned. This patient also required a percutaneous PV balloon venoplasty with stenting, 4 months later.

The postoperative recovery was otherwise uneventful in the majority of patients, and it was characterized by a rapid normalization of their liver tests and function (Figure 1). An anticoagulant and antiaggregant treatment was administered in all of the patients as per the protocol. An antithrombin substitution was started as soon as it was necessary after their LTs. However, because the heparin treatment was only started when their INR was close to normal, many patients initiated their treatment straight away upon their antiaggregant therapy (Figure 2). A heparin anticoagulation treatment was used in only 25 children, starting after a median of 4 days after their surgery (range 0-10 days) and for a duration of around 2 weeks (median: 14 days, range: 3-37). All of the recipients received an antiaggregant treatment. Only two patients (1.6\%) necessitated a reintervention for a hemorrhage within the first month after their LT.

\section{5 | Late follow-up, vascular complications, and their management}

Late vascular complications ( $>30$ days after the LT) were diagnosed in 11 patients. They consisted respectively of the following:

1. One late artery thrombosis was diagnosed at POD 70; it was clinically silent and recovered with a satisfactory development of collateral revascularization; the graft is functioning well and is healthy (normal liver tests and function, 6 years after their LT).

2. One HA stricture was managed successfully by radiological interventional angioplasty at POD 72.

3. PV stenoses in six cases were all managed by radiological interventional angioplasty (median number of dilatations: 2, range: 1-5), with two requiring the positioning of a stent. One of the two patients with a stent developed a subsequent PV thrombosis, and this was cured by a meso-Rex bypass. ${ }^{36}$ All of the grafts and all the patients are doing well.

4. PV thromboses were diagnosed in another three cases (median time for diagnosis: 307 days after their LT), including one who had a right split graft. Of these three, one was cured by a meso-Rex bypass and one is waiting for surgery. The recipient of the right split graft is currently doing well, with an absence of clinical complications.

5. Lastly, the patient with a PV agenesy and with a malformation of the venous abdominal system, who had previously had a portal revascularization of the vena cava, benefited from a conversion into a meso-Rex bypass after the vascular reassessment and when he was over $12 \mathrm{~kg}$ of weight ( 1 year after the LT).

There were no IVC or outflow complications in this series. 


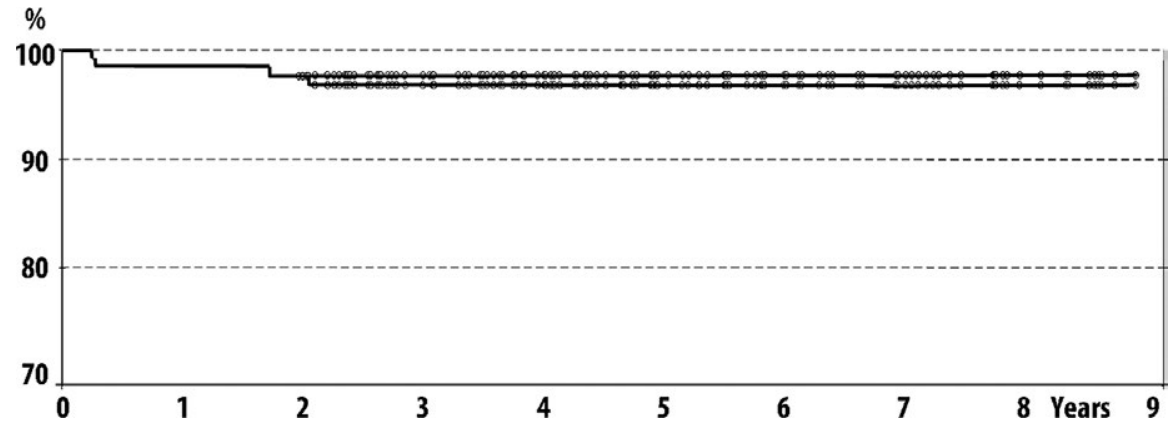

FIGURE 3 The outcomes of 126 first isolated LTs (2008-2015): Kaplan-Meier test for the survival of patients (upper line) and liver grafts (lower line)

\subsection{Clinical outcomes and graft function}

With a median follow-up of 57 months (3-112 months), the actual patient and graft survival rates were $97.6 \%$ and $96.8 \%$, respectively (Figure 3). Three children died: Two from a recurrence of the disease (hemophagocytic lymphohistiocytosis and hepatoblastoma, at three and 25 months after their LT, respectively) and 1 other patient from a multiresistant bacterial sepsis, at POD 94. Another patient was successfully retransplanted for a recurrence of a sclerosing cholangitis ( 25 months after their first LT).

At their last available checkup, all of the patients had a Doppler US which showed a patency of all vascular anastomoses-except for one patient with a late HA thrombosis $(0.8 \%)$ and two patients with a thrombosed PV (one is currently waiting for a meso-Rex bypass). No graft has been lost due to vascular complications in the series. All of the patients who had previously had either a vascular revision, an angioplasty, or a meso-Rex bypass have good flows and wellfunctioning grafts.

Overall, there have been six early biliary problems, all of them being biliary leaks that were managed successfully by a surgical exploration, with one of them dealt with by conservative management. Another five patients presented late biliary anastomotic strictureswith none of them having had any previous vascular problems; all five were treated by percutaneous radiological interventions with a good outcome. Only two patients in the series presented ischemic type biliary lesions: In the first patient, who was transplanted in an emergency (fulminant hepatitis) with a split graft from a suboptimal donor, the cholangiography that was performed a year later showed a diffuse biliary damage, typical of a preservation/reperfusion injury, but with an absence of vascular problems. In the other patient, a biliary perianastomotic stricture (relatively long and requiring a stent) was diagnosed after a late thrombosis of the HA (POD 70); the graft recovered well from both of the complications and is currently functioning, 6 years after the LT, while at the same time, it is displaying normal liver tests.

Of the whole series, 122 patients are surviving at the long term with their first graft. Their liver tests are within the normal range in 96 children (78.7\%) (at a median follow-up of 59.5 (3110) months); they are within the limit of normal to three times the upper normal values in 17 other cases (13.9\%) (at a median follow-up of 60 (6-112) months); only eight children have results above three times the upper normal limit (6.6\%) (at a median follow-up of 34.5 (12-93) months). At the last visit, all of the patients were receiving immunosuppression as follows: tacrolimus in all of them, combined with mycophenolate mofetil in 39 cases, or TOR inhibitors in 10 cases.

A liver biopsy was performed at the long term in 120 patients (a median time after their LT of 25 months, range: 6-93 months): A graft histology was normal in most of the cases, with 10 having features of an acute rejection and 19 patients with signs of some chronic inflammation. Fibrosis was scored systematically on the biopsies, according to the Ishak scoring system; in concordance with the most recent biopsy for each patient, the score was F0 or F1 in $89 \%$ of those with a follow-up of $2-5$ years and in $75 \%$ of the cases with a follow-up $>5$ years after their LT.

\section{5 | DISCUSSION}

The ELTR has collected data and outcomes since 1968 and has accumulated information about 12239 pediatric liver transplants that were performed between 1968 and 2015 (Figure 4). As time has passed and liver transplantation has become of age, the results have improved and a significant improvement has been observed for each period of 5 years, when compared to the previous one, up until 2000 , with $P$ values of 0.003 or lower. Since the year 2000 and for the three consecutive periods of 5 years (2000-2004, 2005-2009, and 2010-2014) there has been no further improvement and the $P$ values in comparing these three periods are $0.14,0.39$, and 0.48 , respectively (Figure 4): The three latter survival curves are following exactly the same pattern over each of the 5 years, suggesting that the transplant community has hit a wall. Although it is clear that this is multifactorial and that many expert centers have achieved better results, at a single-center level, when compared to the median multicenter ELTR value, it points to the fact that new strategies or therapies are needed in order to progress further as a community.

With a similar starting point, Englesbe et $\mathrm{al}^{17}$-a large group of North American transplant centers-have recently contributed to an initiative within the SPLIT, developing an innovative methodology that has ended by proposing a novel broad-based quality improvement proposal. Interestingly, by focusing on the variation in outcomes across the transplant centers (a 20\% differential in 1-year survival), they have concentrated their interest on studying the practices that are associated with lower incidences of $\mathrm{HA}$ thrombosis and 
FIGURE 4 The ELTR: pediatric patient survival vs the period of liver transplantation (1968 -2016). Global log rank $P<0.0001$. Log rank $P=N S$, for comparing each of the periods 2000 2004, 2005-2009, and 2010-2014

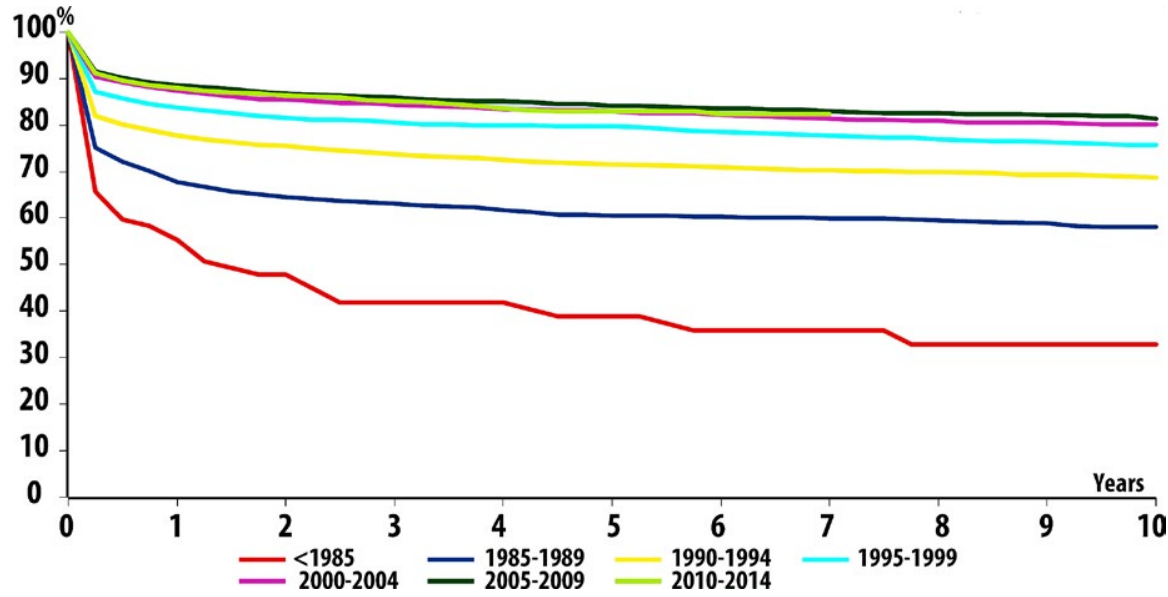

biliary complications-which are considered the main surgical issues. Although they have mentioned that quality improvement plans for transplantation cannot target only the technical aspects and problems (as solving only these aspects would not be sufficient to bring the median outcome over 95\%), they have brought an attention to the need for technical refinements, as a road map forward within the transplantation community.

Not surprisingly, many centers around the world, including our center, have developed independently of the SPLIT initiative, a surgical strategy, and a management approach that are very similar to the "best practice report" as proposed by Englesbe et al ${ }^{17}$ for the prevention of HA thrombosis. After their report in 2012, the SPLIT group has not reported any further about the success, or the failure, of the best practice and of the quality improvement plan. However, the results of this series and of others in the literature seem to confirm that their approach was adequate and that such a road map should be disseminated and practiced with a more standardized procedure.

Vascular complications as a leading cause of graft and patient loss after an LT have been evidenced in many series, with an incidence of up to $25 \% .{ }^{1-6}$ The incidence is higher in children than when compared to adults, very likely due to specific issues, such as a low recipient weight, a large donor/recipient mismatch, and other anatomical factors, such as a PV hypoplasia. ${ }^{7-13,20}$ Not surprisingly, by reducing the incidence of these complications, and/ or by minimizing their damaging effects on the graft by an early diagnosis and by a rapid adequate management, these actions have a major impact on the outcome-not only in the short term, but also in the very long term. It is likely that even though a graft may tolerate some ischemic damage and survive, the related sequelae may occur secondarily and will only become evident with time and the limit of the life span of the graft. A late PV thrombosis is a good example of an initially well-tolerated problem, but it causes extrahepatic portal hypertension that can last for many years without clinical complications; generally, it brings the patient to a very difficult condition with exceedingly challenging retransplantation; an early meso-Rex bypass has been shown to be a valid option to manage these cases. ${ }^{36-38}$
This cohort of patients has allowed for drawing some robust conclusions. The series was large and homogenous, and the follow-up was long enough for all of the grafts. Interestingly, more than $80 \%$ of the liver grafts were technical variants, which is typical of a pediatric series but uncommon in many centers. Split grafts procured from postmortem donors represented $50 \%$ of the transplanted grafts, with another $30 \%$ of all grafts procured from living donors. The latter procedure was introduced in the fourth year of the center experience, at a time when split liver graft offer from optimal donors was decreasing in number, with the collateral effects of prolonged waiting time and clinical deterioration of the candidates while waiting for the transplant. However, the latter problem was addressed by the transplant national authorities in 2015 with a new policy (obligatory split) taking effect in late 2015; this augmented dramatically the split graft offer (from 32 to 54 split annually): at that time, and by 2016 , the living program was revisited with a drop of the numbers.

All of the transplants were performed over a relatively short time span (7 years) by a single team, using all of the same standardized operative strategies and techniques, as well as by employing those stated protocols for both perioperative management and immunosuppression. Interestingly, the surgical approaches that were applied for these patients were a combination of what have been recommended by different teams over the last decade, for instance, the use of microsurgical techniques, PV hypoplasia corrections, reducing the use of arterial vascular grafts, multiple USD checks during and after the LTs, and an early diagnosis and a rapid aggressive management for any vascular problem, among others. ${ }^{15,16,18-24,28,31,32}$ With a zero incidence of graft loss related to vascular problems and with only two of the 122 currently functioning grafts having a non-clinically relevant vascular sequela, it can be concluded that the strategy was instrumental in achieving the excellent outcomes. It was close to a $98 \%$ actual patient survival over a 7-year period-and with a minimum follow-up of 2 years afterward for each graft. These results compare well with other series that have been published recently ${ }^{6,7,39-43}$ or even earlier. ${ }^{6,44-46}$ More interestingly, these results have suggested that the vast majority of surviving grafts have an excellent function at the very long-term point, with fully normal liver tests in $78.7 \%$ of the patients. At that 
long-term point, with a low fibrosis score (F0 or F1) at biopsy in $>80 \%$ of the whole cohort, this compares well with other series. ${ }^{47}$ The latter remarks are forceful elements suggesting that the strategies, comprehensively, have had a protective effect-in fact, more than simply targeting at an improved early clinical period.

In this series, all of the vascular flow abnormalities that were detected by the USD test during surgery were managed extemporaneously: The flows were checked as being normal in all of the vessels at the end of the procedure-before the patient was moved back into the ICU. The study believes that the latter actions were an important aspect of the general strategy and that they were instrumental in achieving the good outcomes in this cohort. Postoperative vascular complications occurred in 19 cases overall (15\%), including minor events. They were all successfully cured-with the exception of one late HA thrombosis and one late PV thrombosis with a right split graft. All of the grafts, excluding the 2 latter ones, are healthy and functioning well, with an absence of clinical complications. The actual graft survival rate was $96.8 \%$, with 119 of the 122 surviving grafts $(97.5 \%)$ having a vascular anastomosis patency at the USD test.

Few teams have proposed implementing a relatively aggressive anticoagulation protocol after an $\mathrm{LT},{ }^{17,18,22,33}$ starting very early after the graft reperfusion and aiming at higher levels of anticoagulation. This study's data have shown, however, that a full clinical anticoagulation status was not necessary to efficiently prevent thrombosis during the first weeks after an LT, which may help in reducing the risk of postoperative bleeding or the need for transfusions or reintervention for a hemorrhage. These experiences would suggest that overall, both the surgical refinements and the role of radiological follow-up and interventions must be equally emphasized and that an anticoagulation therapy has a complementary role, not a solution for all problems.

\section{CONCLUSION}

Overall, vascular complications after a pediatric LT are the leading cause of graft loss and mortality. An increasing attention has been given by many teams in the last decade, in order to prevent and best manage these complications, in order to avoid or limit any ischemic damage and sequelae. This series has confirmed previous reports and other series suggesting that the strategy must be multifaceted. Although a zero event incidence may remain difficult to reach, it is possible to significantly reduce the related graft loss, both in the short term and in the long term. Also, it significantly impacts on patient survival and the retransplantation rate.

The results highlight that there must be an attention to technical details at surgery, a balanced anticoagulation therapy to avoid the hypercoagulability state, and also an aggressive policy of repeated USD tests, combined with a zero tolerance of flow abnormalities-thus a preemptive management of vascular flow abnormalities after an LT, as well as the timely cure of late vascular strictures or thrombosis whenever feasible. Altogether, this seems to be an effective and successful strategy in order to limit graft and patient loss.

\section{ACKNOWLEDGMENTS}

The authors would like to Thank Vincent Karam and Valérie Cailliez from the ELTR for their contribution in allowing for the publication of Figure 4 and the related statistical results. ELTR is supported by a grant from Astellas, Novartis, Institut Georges Lopez, and Bridge to Life and logistic support from the Paul Brousse Hospital (Assistance Publique-Hopitaux de Paris).

\section{AUTHORS' CONTRIBUTIONS}

Chiara Grimaldi: took the lead in designing the study and the related analysis, and in writing drafts and the final manuscript; Fabrizio di Francesco, Fabrizio Chiusolo, and Lidia Monti: contributed to material and data acquisition; Roberta Angelico: contributed to the data collection, statistical analysis, interpretation of the results, and preparation of the manuscript; Jean de Ville de Goyet: devised the main conceptual ideas and directed the project and proof outline; and all authors: provided critical feedback and helped finalizing the manuscript.

\section{ORCID}

Fabrizio Chiusolo (D) http://orcid.org/0000-0003-0265-609X

Jean de Ville de Goyet (iD http://orcid.org/0000-0001-7681-6178

\section{REFERENCES}

1. McDiarmid SV, Anand R, Martz K, Millis MJ, Mazariegos G. A multivariate analysis of pre-, peri-, and post-transplant factors affecting outcome after pediatric liver transplantation. Ann Surg. 2011;254:145-154.

2. Herden U, Ganschow R, Grabhorn E, Briem-Richter A, Nashan B, Fischer L. Outcome of liver re-transplantation in children-Impact and special analysis of early re-transplantation. Pediatr Transplant. 2014;18:377-384.

3. Davis A, Rosenthal P, David Glidden D. Pediatric liver retransplantation: outcomes and a prognostic scoring tool. Liver Transpl. 2009;15:199-207.

4. Sieders E, Peeters PMJG, TenVergert EM, et al. Early vascular complications after pediatric liver transplantation. Liver Transpl. 2000;6:326-332.

5. Ng V, Anand R, Martz K, Fecteau A. Liver retransplantation in children: a SPLIT database analysis of outcome and predictive factors for survival. Am J Transplant. 2008;8:386-395.

6. Spada M, Riva S, Maggiore G, Cintorino D, Gridelli B. Pediatric liver transplantation. World J Gastroenterol. 2009;15:648-674.

7. Kasahara M, Umeshita K, Sakamoto S, Fukuda A, Furukawa H, Uemoto S. Liver transplantation for biliary atresia: a systematic review. Pediatr Surg Int. 2017;33:1289-1295.

8. Arnon R, Annunziato R, Miloh T, et al. Liver transplantation in children weighing $5 \mathrm{~kg}$ or less: analysis of the UNOS database. Pediatr Transplant. 2011;15:650-658.

9. Noujaim HM, Mayer DA, Buckles JA, et al. Techniques for and outcome of liver transplantation in neonates and infants weighing up to 5 kilograms. J Pediatr Surg. 2002;37:159-164.

10. Heffron TG, Pillen T, Smallwood G, et al. Incidence, impact and treatment of portal and hepatic venous complications following pediatric liver transplantation: a single center 12 years experience. Pediatr Transplant. 2010;14:722-729. 
11. Neto JS, Fonseca EA, Feier FH, et al. Analysis of factors associated with portal vein thrombosis in pediatric living donor liver transplant recipients. Liver Transpl. 2014;20(10):1157-1167.

12. Farmer DG, Venick RS, McDiarmid SV, et al. Predictors of outcomes after pediatric liver transplantation: an analysis of more than 800 cases performed at a single institution. J Am Coll Surg. 2007;204:904-914.

13. Yilmaz A, Arikan C, Tumgor G, Kilic M, Aydogdu S. Vascular complications in living related and deceased donation pediatric liver transplantation: single center experience from Turkey. Pediatr Transplant. 2007;11:160-164.

14. Kasahara M, Morioka D, Sakamoto S, et al. Vascular reconstruction and complications in living donor liver transplantation in infants weighting less than 6 kilograms: the Kyoto experience. Liver Transpl. 2006;12:1224-1232.

15. Ackermann O, Branchereau S, Franchi-Abella S, et al. The longterm outcome of hepatic artery thrombosis after liver transplantation in children: role of urgent revascularization. Am J Transplant. 2012;12:1496-1503.

16. Abdelaziz $\mathrm{O}$, Attia $\mathrm{H}$. Doppler ultrasonography in living donor liver transplantation recipients: intra- and post-operative vascular complications. World J Gastroenterol. 2016;22:6145-6172.

17. Englesbe MJ, Kelly B, Goss J, et al. Reducing pediatric liver transplant complications: a potential roadmap for transplant quality improvement initiatives within North America. Am J Transplant. 2012;12:2301-2306.

18. Hardikar W, Poddar U, Chamberlain J, et al. Evaluation of a postoperative thrombin inhibitor replacement protocol to reduce haemorrhagic and thrombotic complications after paediatric liver transplantation. Thromb Res. 2010;126:191-194.

19. Ren X, Guan J, Gao N, Niu H, Tang J. Evaluation of pediatric liver transplantation-related artery complications using intra-operative multi-parameterultrasonography. MedSciMonit. 2016;22:4495-4502.

20. de Magnée C, Bourdeaux C, De Dobbeleer F, et al. Impact of pre transplant hemodynamics and porta reconstruction techniques on post transplant portal vein complications in pediatric liver transplantation: a retrospective analysis of 197 recipients. Ann Surg. 2011;254:55-61.

21. Herrero A, Souche R, Joly E, et al. Early hepatic artery thrombosis after liver transplantation: what is the impact of the arterial reconstruction type? World J Surg. 2017;41(8):2101-2110.

22. Ziaziaris WA, Darani A, Holland AJA, et al. Reducing the incidence of hepatic artery thrombosis in pediatric LT: effect of microvascular techniques and a customized anticoagulation protocol. Pediatr Transplant. 2017;21. https://doi.org/10.1111/petr.12917

23. Sommovilla J, Doyle MM, Vachharajani $\mathrm{N}$, et al. Hepatic venous outflow obstruction in pediatric liver transplantation: technical considerations in prevention, diagnosis, and management. Pediatr Transplant. 2014;18:497-502.

24. Tannuri U, Santos MM, Tannuri AC, et al. Which is the best technique for hepatic venous reconstruction in pediatric living donor liver transplantation? experience from a single center. J Pediatr Surg. 2011;46:1379-1384.

25. de Ville de Goyet J, Struye de Swielande Y, Reding R, Sokal EM, Otte JB. Delayed primary closure of the abdominal wall after cadaveric and living related donor liver graft transplantation in children: a safe and useful technique. Transpl Int. 1998;11(2):117-122.

26. Sheth J, Sharif K, Lloyd C, et al. Staged abdominal closure after small bowel or multivisceral transplantation. Pediatr Transplant. 2012;16:36-40.

27. García-Criado A, Gilabert R, Nicolau C, et al. Early detection of hepatic artery thrombosis after liver transplantation by Doppler ultrasonography: prognostic implications. J Ultrasound Med. 2001;20:51-58.

28. Uchida Y, Sakamoto S, Egawa H, et al. The impact of meticulous management for hepatic artery thrombosis on long-term outcome after pediatric living donor liver transplantation. Clin Transplant. 2009;23:392-399.
29. Kaneko J, Sugawara Y, Akamatsu N, et al. Prediction of hepatic artery thrombosis by protocol Doppler ultrasonography in pediatric living donor liver transplantation. Abdom Imaging. 2004;29:603-605.

30. Ignjatovic V, Furmedge J, Newall F, et al. Age-related differences in heparin response. Thromb Res. 2006;118:741-745.

31. Uslu N, Aslan H, Tore HG, et al. Doppler ultrasonography findings of splenic arterial steal syndrome after liver transplant. Exp Clin Transplant. 2012;10:363-367.

32. Gu LH, Fang H, Li FH, et al. Prediction of early hepatic artery thrombosis by intraoperative color Doppler ultrasound in pediatric segmental liver transplantation. Clin Transplant. 2012;26:571-576.

33. Arshad F, Lisman T, Porte RJ. Hypercoagulability as a contributor to thrombotic complications in the liver transplant recipient. Liver Int. 2013;33:820-827.

34. Ishak K, Baptista A, Bianchi L, et al. Histological grading and staging of chronic hepatitis. J Hepatol. 1995;22:696-699.

35. Pinto S, Reddy SN, Horrow MM, Ortiz J. Splenic Artery Syndrome after orthotopic liver transplantation: a review. Int J Surg. 2014;12:1228-1234.

36. de Ville de Goyet J, Lo Zupone C, Grimaldi C, et al. Meso-Rex bypass as an alternative technique for portal vein reconstruction at or after liver transplantation in children: review and perspectives. Pediatr Transplant. 2013;17:19-26.

37. Alvarez F. Portal vein complications after pediatric liver transplantation. Curr Gastroenterol Rep. 2012;14:270-274.

38. de Ville de Goyet J, Clapuyt P, Otte JB. Extrahilar mesenterico-left portal shunt to relieve extrahepatic portal hypertension after partial liver transplant. Transplantation. 1992;53:231-232.

39. Otte JB. Pediatric liver transplantation: personal perspectives on historical achievements and future challenges. Liver Transpl. 2016;22:1284-1294.

40. Nishimura N, Kasahara M, Ishikura K, Nakagawa S. Currentstatus of pediatric transplantation in Japan. J Intensive Care. 2017;5:48-57.

41. Neto JS, Feier FH, Bierrenbach AL, et al. Impact of Kasai portoenterostomy on liver transplantation outcomes: a retrospective cohort study of 347 children with biliary atresia. Liver Transpl. 2015;21:922-927.

42. Wan P, Xu D, Zhang J, et al. Liver transplantation for biliary atresia: a nationwide investigation from 1996 to 2013 in mainland China. Pediatr Transplant. 2016;20:1051-1059.

43. Safwan M, Ramachandran P, Reddy MS, SHamugam N, Rela M. Living donor liver transplantation for biliary atresia-an Indian experience. Pediatr Transplant. 2016;20:1045-1050.

44. Chen CL, Concejero A, Wang CC, et al. Living donor liver transplantation for biliary atresia: a single-center experience with first 100 cases. Am J Transplant. 2006;6:2672-2679.

45. Utterson EC, Shepherd RW, Sokol RJ, et al. Biliary atresia: clinical profiles, risk factors, and outcomes of 755 patients listed for liver transplantation. J Pediatr. 2005;147:180-185.

46. Fouquet $\mathrm{V}$, Alves $\mathrm{A}, \mathrm{Branchereau} \mathrm{S}$, et al. Long-term outcome of pediatric liver transplantation for biliary atresia: a 10-year follow-up in a single center. Liver Transpl. 2005;11:152-160.

47. Venturi C, Sempoux C, Bueno J, et al. Novel histologic scoring system for long-term allograft fibrosis after liver transplantation in children. Am J Transplant. 2012;12:2986-2996.

How to cite this article: Grimaldi C, di Francesco F, Chiusolo F, et al. Aggressive prevention and preemptive management of vascular complications after pediatric liver transplantation: $\mathrm{A}$ major impact on graft survival and long-term outcome. Pediatr Transplant. 2018;e13288. https://doi.org/10.1111/petr.13288 